LA-URA-98- $3-3585$

Title:

$$
\text { CONF-9805/67- }
$$

HISTONE-DNA CONTACTS IN STRUCTURE/FUNCTION RELATIONSHIPS OF NUCLEOSOMES AS REVEALED BY CROSSLINKING

Author(s):

Submitted to:

E. Morton Bradbury, Life Sciences Division, MS M888, Los Alamos National Laboratory, Los Alamos, NM 87545 USA and Dept. of Biological Chemistry, School of Medicine, University of California, Davis, CA 95616 USA

Sergei I. Usachenko, Department of Biological Chemistry School of Medicine, University of California, Davis, CA 95616 USA

Structural and Functional Genomics

Trieste, Italy

May 4-8, 1998

DISTRIBUTION OF THIS DOCUMENT IS UNLAMTED

APR 131999
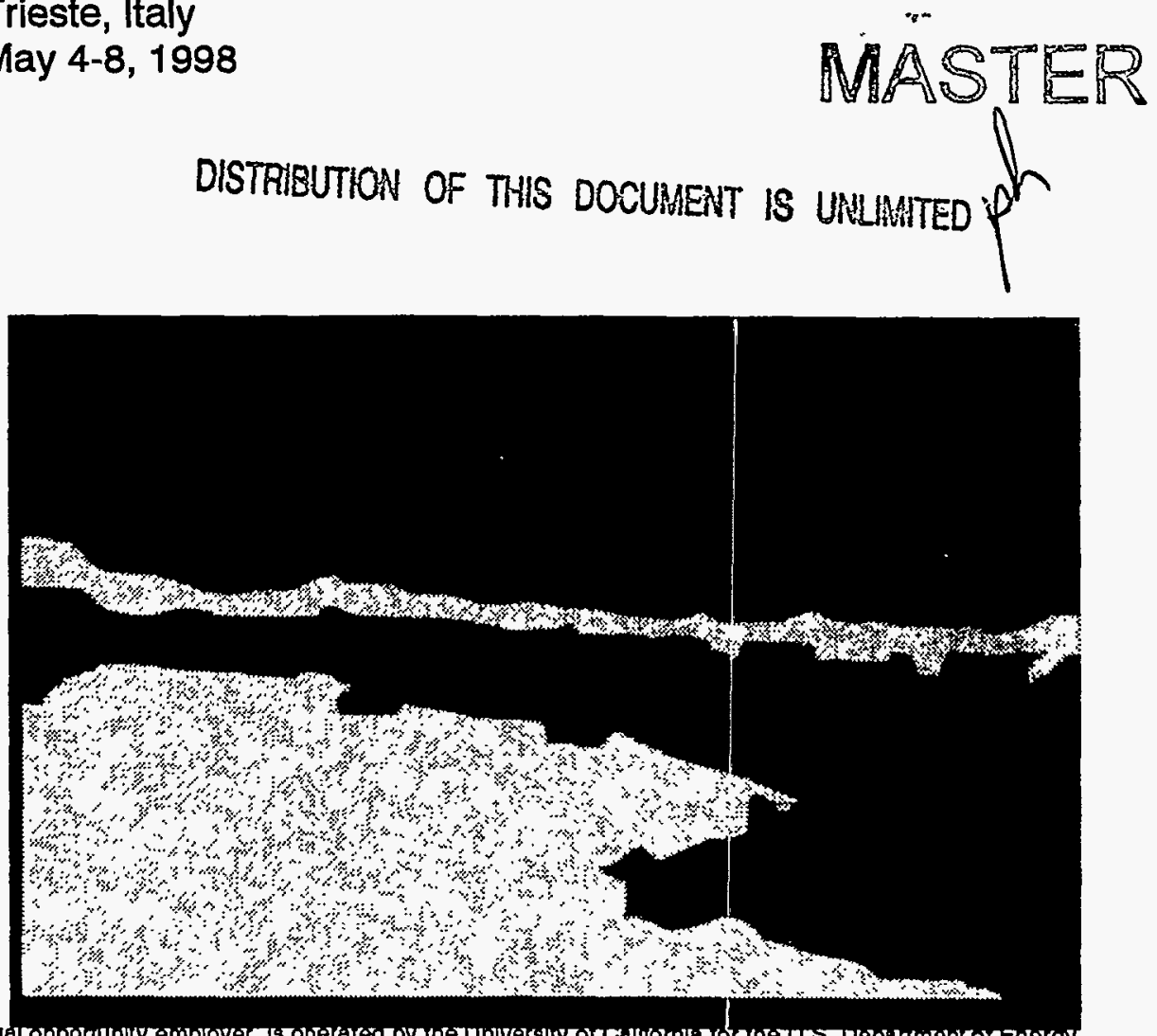

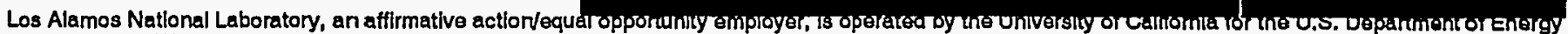
under contract W-7405-ENG-36. By acceptance of this article, the publisher recognizes that the U.S. Government retains a nonexclusive, royaity-frea Iconse to publish or reproduce the published form of this contributlon, or to allow others to dó so, for U.S. Governiment purposes. Thë [os Aläniós Nationaltzabiotatory requests that the publlsher Identify this artlcle as work performed under the ausplces of the U.S. Department of Energy. The Los Alamos National Laboratory strongly supports academic treedom and a researcher's right to publish; as an institutlon, however, the Laboratory does not endorse the viewpoint of a publicatlon or guarantee its technical correctness. 


\section{DISCLAIMER}

This report was prepared as an account of work sponsored by an agency of the United States Government. Neither the United States Government nor any agency thereof, nor any of their employees, makes any warranty, express or implied, of assumes any legal liability or responsibility for the accuracy, completeress, or usefulness of any information, apparatus, produce, or process disclosed, or represents that its use would not infringe privately owned rights. Reference herein to any speciric commercial product, process, or service by trade name, trademarik, inanufacturer, or otherwise does not necessarily constitute or imply its endorsement, recommendation, or favoring by the United States Goyernment or any agency thereof. The views and opinions of authors expressed herein do not necessarily state or reffect those of the United States Goverameat or any agency thereof. 


\section{DISCLAIMER}

Portions of this document may be illegible in electronic image products. Images are produced from the best available original document. 


\title{
HISTONE-DNA CONTACTS IN STRUCTURE/FUNCTION RELATIONSHIPS OF NUCLEOSOMES AS REVEALED BY CROSSLINKING
}

\author{
SERGEI I. USACHENKO \\ Department of Biological Chemistry \\ School of Medicine \\ University of California \\ Davis, CA 95616 \\ USA \\ E. MORTON BRADBURY \\ Life Science Division \\ Los Alamos National Laboratory \\ MS M888 \\ Los Alamos, New Mexico 87545 \\ USA \\ Department of Biological Chemistry \\ School of Medicine \\ University of California \\ Davis, CA 95616 \\ USA
}

The magnitude of the problem of understanding the structure/function relationships of eukaryotic chromosomes can be appreciated from the fact that the human diploid genome contains more than 2 meters of DNA packaged into 46 chromosomes, each at metaphase being several microns in length. Each chromatid of a chromosome contains a single DNA molecule several centimeters in length. In addition to the DNA, chromosomes contain an equal weight of histones and an equal weight of non-histone chromosomal proteins. The histones are the major chromosomal structural proteins. The non-histone chromosomal proteins are involved in the DNA processes of transcription and replication, in chromosome organization and in nuclear architecture. Polytene chromosomes with their bands and interbands and puffs of active genetic loci provide visual evidence for long range order as do the bands and interbands of mammalian metaphase chromosomes. The gentle removal of histones and all but the most tightly bound 2-3\% of non-histone proteins from metaphase chromosomes revealed by electron microscopy a residual protein scaffold constraining a halo of DNA loops extending out from the scaffold. Froma variety of studies the loop sizes were found to be in a range from 5 to $200 \mathrm{kbp}$ with an average size of $50 \mathrm{kbp}$ [1]. Thus the haploid genome of 
$3 \times 10^{9}$ bp DNA would contain about 60,000 average size loops, a number comparable with the estimated number of genes in the human genome of 50,000 to 80,000. These observations led to the proposed model of DNA loops packaged by histones into chromatin domains, each domain being regarded as both a structural unit and a genetic unit of chromosomes.

\section{Histones}

Based on their sequences, conformational behavior's and interactions histones fall into three groups; the families of very lysine rich $\mathrm{H1}, \mathrm{H}^{\circ}, \mathrm{H} 5$ etc; the $\mathrm{H} 2 \mathrm{~A}, \mathrm{H} 2 \mathrm{~B}$ and $\mathrm{H} 3$ protein families and $\mathrm{H} 4$. The central importance of histones $\mathrm{H} 3$ and $\mathrm{H} 4$ in chromosome structure and functions is implied by the rigid conservation of their sequences. Histones are multidomain proteins [2]; $\mathrm{H} 1$ has a central structured domain and extended basic $\mathrm{N}$ terminal (1-40 amino acid residues) and C-terminal (120-210 amino acid residues) domains; $\mathrm{H} 2 \mathrm{~A}$ and $\mathrm{H} 2 \mathrm{~B}$ have central structured domains and extended basic $\mathrm{N}$ - and $\mathrm{C}$ terminal domains. Both $\mathrm{H} 3$ and $\mathrm{H} 4$ have a basic, flexible $\mathrm{N}$-terminal domain and a structured central and C-terminal domain. Histones are subjected to cell cycle dependent posttranslational modifications that are strictly associated with chromosome functions $[2$, 3]. These are: i) phosphorylation of $\mathrm{H} 1, \mathrm{H} 2 \mathrm{~A}$ and $\mathrm{H} 3$, ii) acetylation of the core histones $\mathrm{H} 2 \mathrm{~A}, \mathrm{H} 2 \mathrm{~B}, \mathrm{H} 3$ and $\mathrm{H} 4$ and iii) ubiquitination of H2A and H2B. $\mathrm{H} 1$ and $\mathrm{H} 3$ phosphorylations are associated with chromosome condensation; acetylations of the core histones are associated with the DNA processes of replication and transcription. So far the functions of the ubiquitination of $\mathrm{H} 2 \mathrm{~A}$ and $\mathrm{H} 2 \mathrm{~B}$ are unknown. The crystal structure of the histone octamer has been solved to $0.33 \mathrm{~nm}$ resolution [4]. This structure shows clearly the modes of interactions between the central structured domains that stabilize the histone octamer. However electron densities are not observed for the $\mathrm{N}$ - and $\mathrm{C}$-terminal flexible domains. This is attributed to static or dynamic disorder of these regions in the crystal.

\section{Nucleosome Structure}

Virtually all of genomic DNA is packaged into nucleosomes in chromatin. For most somatic cells the nucleosome contains $195 \pm 10$ bp DNA; the histone octamer (H2A, $\mathrm{H} 2 \mathrm{~B}, \mathrm{H} 3, \mathrm{H} 4)_{2}$ and a very lysine rich histone (e.g. $\mathrm{H} 1, \mathrm{H}^{\circ}, \mathrm{H} 5$ ) also called linker histones. Some nucleosomes from specialized cells contain longer DNA repeats e.g. chicken erythrocytes (212 bp DNA) [3]. The ends of the nucleosomal DNA can be trimmed by micrococcal nuclease digestion to give two well-defined sub-nucleosome particles. These are the chromatosome containing 168 bp DNA, the histone octamer and a linker histone and the nucleosome core particle containing $146 \pm 1$ bp DNA and the histone octamer. The core particle has been subjected to intensive structural studies. Neutron scatter studies of the core particle in solution proved that DNA was coiled on the outside of the histone octamer [5]. Low resolution x-ray diffraction (2 $\mathrm{nm})$ [6] and 
neutron diffraction $(1.6 \mathrm{~nm})$ [7] gave structures in agreement with the neutron scatter solution structure. In the crystal structure of the core particle at $0.7 \mathrm{~nm}$ resolution, DNA was found not to coil uniformly around the histone octamer but to follow a more irregular path with DNA bends [8]. Very recently the crystal structure of the core particle has been solved to $2.8 \AA$ resolution [9]. At this higher resolution, the DNA bends are found to be complexed with clusters of basic residues. The structure of the histone octamer in the core particle is virtually identical to that reported for the isolated octamer [4]. In the crystal structure of the core particle, the basic N-terminal domain of histone $\mathrm{H} 4$ is observed binding to the ( $\mathrm{H} 2 \mathrm{~A}, \mathrm{H} 2 \mathrm{~B})$ dimer of the adjacent core particle [9]. It is not known if this is an intranucleosome native binding site or the result of a rearrangement of flexible histone terminal domains on removal of linker DNA to give the core particle. Parts of the $\mathrm{N}$-terminal domains of the other histones are observed between the gyres of the DNA coil. However, little or no electron density was observed for the N-terminal domains outside of the core particle. Previously it was shown that the calculated radius of gyration $(\mathrm{Rg})$ of the histone octamer electron density was $2.97 \mathrm{~nm}$ compared to the measured value of $3.3 \mathrm{~nm}$ from neutron scatter [10]. Controlled proteolytic removal of histone terminal domains from the octamer reduced its $\mathrm{Rg}$ from 3.35 to $2.98 \mathrm{~nm}$. Clearly, these flexible terminal domains are accessible to proteases as they must be to kinases, acetyltransferases, deacetylases and ubiquitinases. These disordered histone terminal domains correspond exactly to the mobile regions identified by nuclear magnetic resonance spectroscopy studies of the histone octamer [11]. An ultimate understanding of histone functions in chromosomes will require detailed knowledge of the conformational behaviors of the terminal domains, their sites of interactions in chromatin and the effects of reversible chemical modifications on these interactions. This will be a formidable task because there is no functional assay and in in vitro studies these flexible histone.terminal domains may rearrange. It is essential that the native binding sites of these histone regions be determined in nuclei and at different phases of the cell cycle. One of the most powerful and informative methods for this task is zero-length covalent protein-DNA crosslinking induced by mild methylation of DNA with dimethylsulfate [12].

\section{Histone-DNA Crosslinking}

This method allows the direct determination of certain histone-DNA contacts in nucleosomes and is particularly suited for studies of histone-DNA interactions both in vitro $[13,14]$ and in vivo $[15,16]$. In addition, this is the only methodology which provides a direct approach for the localization of specific histone-DNA contacts in chromosomes within intact cell nuclei $[13,17]$. There are two protocols used in this methodology [18]. The first protocol, most commonly used to study histone-DNA contacts in nucleosomes, favors crosslinking through histidines ( $90 \%)$ rather than lysines $(-10 \%)$, whereas the second protocol provides crosslinking of histones through both histidines $(\sim 60 \%)$ and lysines $(-40 \%)$. The method and two-dimensional gel- 
electrophoresis for the mapping of crosslinking sites on the nucleosomal DNA are shown in the Figures $I$ and 2 respectively.

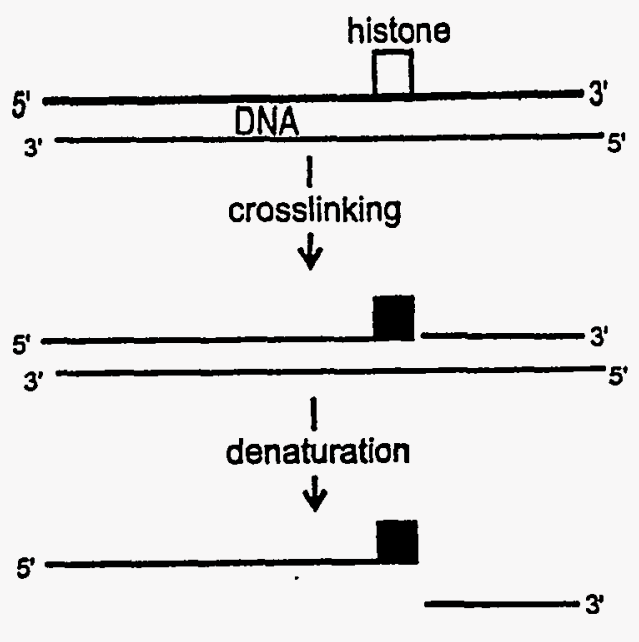

Figure 1. The principle scheme of histone-DNA crosslinking induced by mild methylation of DNA with dimethyl sulfate. Crosslinking causes a single stranded nick in the nucleosomal DNA at the crosslinking site such that only the 5 -terminal DNA fragment is crosslinked to a histone molecule. The length of the crosslinked DNA fragment is the precise distance of a protein crosslinking site from the 5 -end of the nucleosomal DNA and can be assessed by using two systems of twodimensional gel electrophoresis.

\section{Core Histone-DNA Contacts in Nuclei Isolated from Different Sources}

Using this methodology histone-DNA contacts were first determined in chromatin and nucleosomes isolated from different sources $[19,20]$. Further improvement of this method allowed the determination of the arrangement of histones on nucleosomal DNA in intact nuclei isolated from different kingdoms (animals, plants and yeast) [17]. Although these particles represented a highly heterogeneous population that differ in histone modification, histone variants and nonhistone proteins, the primary organization (linear sequential arrangement of histone-DNA contacts along nucleosomal DNA) of nucleosomal cores in all eukaryotes was found to be invariable [17]. The main difference in histone subtypes provided a significant improvement in the resolution of the diagonals corresponding to individual histones in the 2D gel electrophoresis (Fig.3 $A, B)$. This allowed a more defined map of histone-DNA contacts in the nucleosome to 

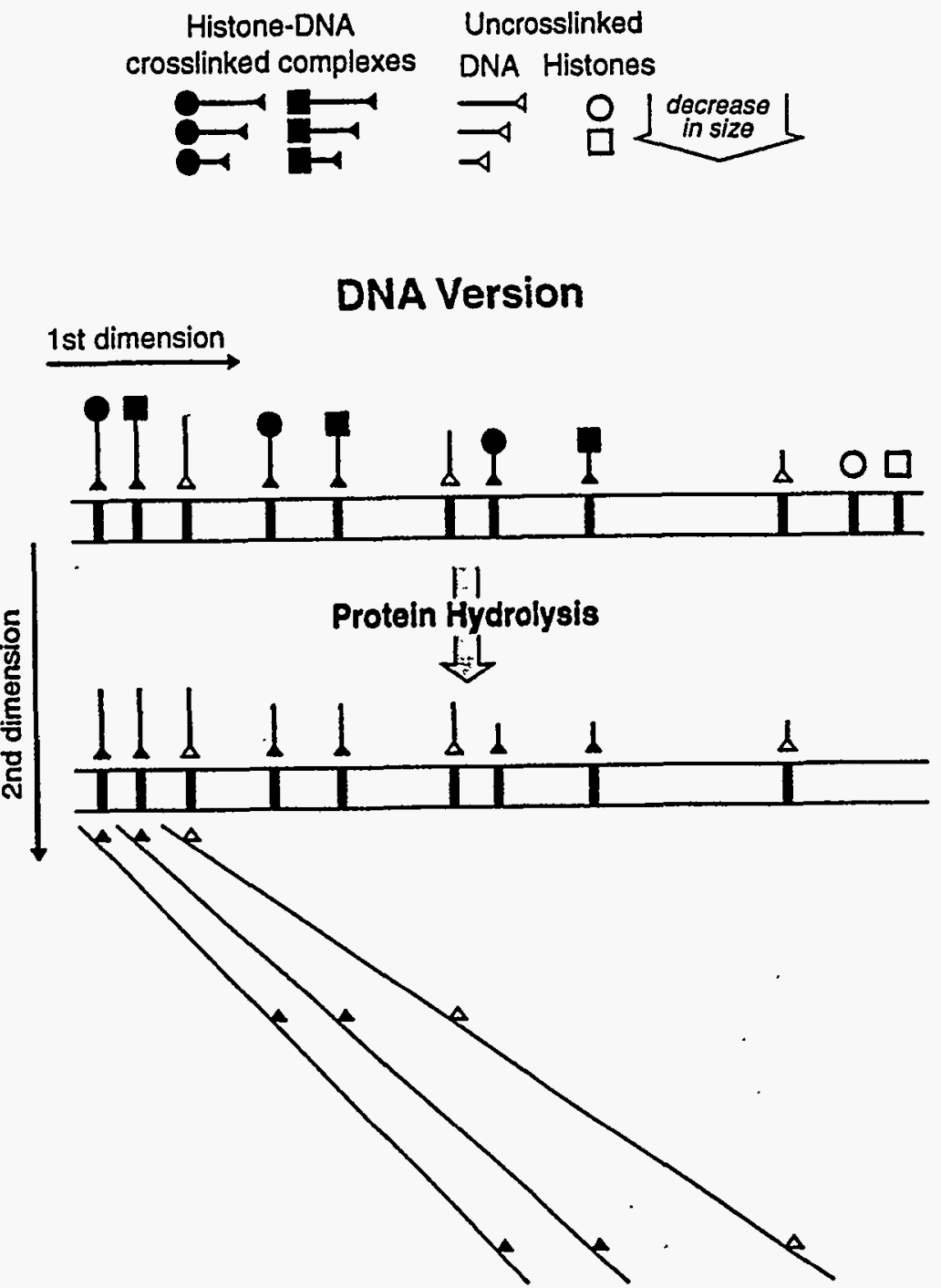


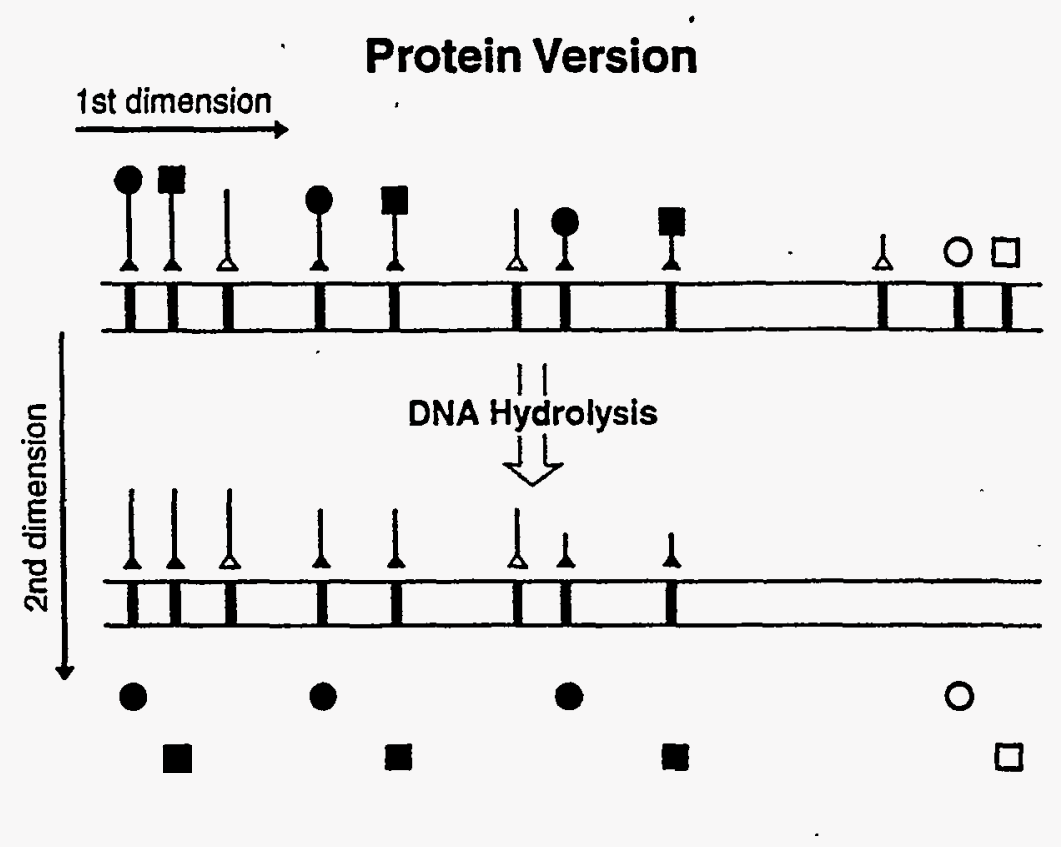

Figure 2. Schematic presentation of two versions of two-dimensional gel electrophoresis. In the first dimension which is the same for both versions, the mobility of the crosslinked histone-DNA complexes in the denaturing SDS gel depends on the molecular weight of the histone and the size of the crosslinked DNA fragment. In the "DNA version", after separation of histone-DNA crosslinked complexes in the first dimension the histones are digested directly in the gel by Protease and the released ${ }^{32} \mathrm{P}$-labeled DNA fragments are separated according to their size in a denaturing second dimension gel. In the first dimension, histones crosslinked to the DNA decrease the mobility of the crosslinked complexes proportionally to the histone size. As a resulh, after histone digestion, the released DNA fragments that were crosslinked to the different histones fall on different diagonals in the second dimension gel. The diagonals which correspond to particular histones in the twodimensional gel are arranged from left to right in the same order as free histones migrating from top to bottom in the one-dimensional SDS giel. The position of the spots within each diagonal indicates the length of the DNA fragments crosslinked to a particular histone. This length can be determined by running DNA fragments of known size in the gel of the second dimension.

in the "Protein version", after first dimension DNA in the crosslinked complexes is chemically degraded directly in the gel and the released ${ }^{125}$ I-labeled histones are then separated in the second dimension SDS gel according to their size. In the first dimension, the mobility of the crosslinked complexes depends on the histones' molecular weight and the size of the crosslinked DNA fragment. As a result, in the gel of the second dimension, the released histones are arranged as spots on different horizontal lines. The position of these spots on a particular horizontal line indicates the location of DNA crosslinking sites for a particular histone from the 5 'end of the nucleosomal DNA. This location can be determined by measuring the length of a single stranded.DNA fragment crosslinked to the histone according to the difference in mobility between the crosslinked complex and the noncrosslinked protein in the first dimension. 


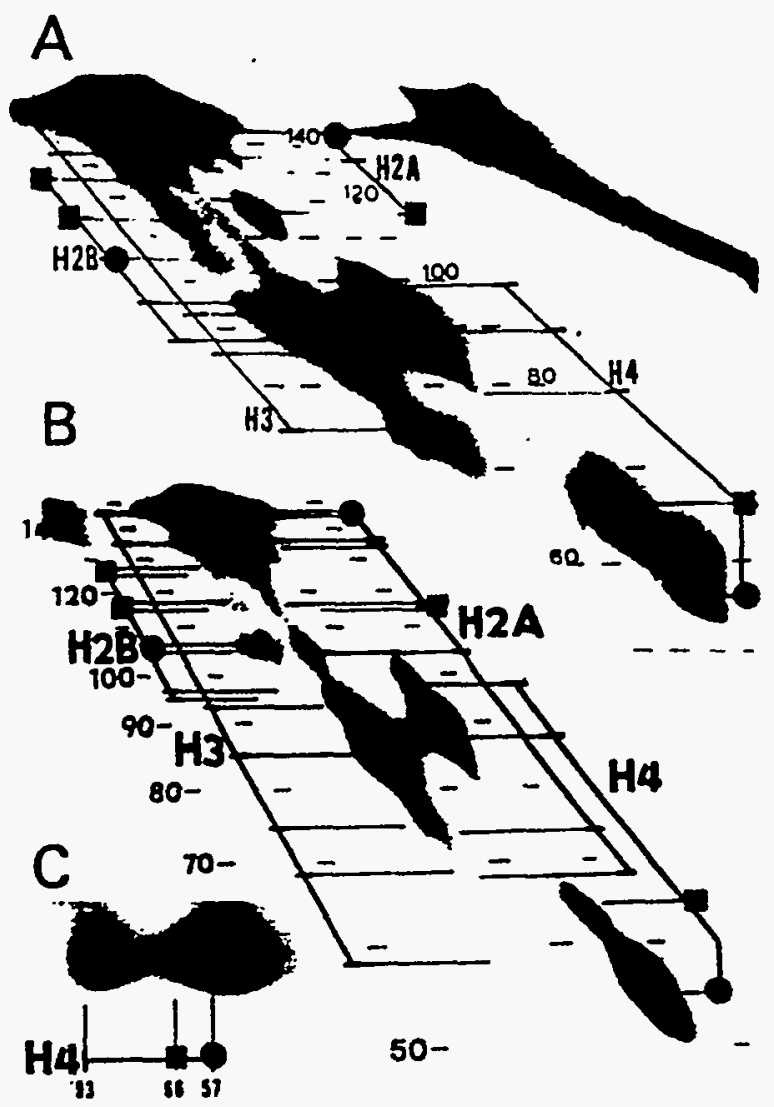

Figure 3. Histone-DNA contacts in the nucleosomal core in nuclei from sea urchin sperm (A), lily buds (B), and yeast (C). A and B, "DNA version" of two-dimensional gel electrophoresis of single-stranded, ${ }^{32} \mathrm{P}$. labeled DNA fragments crosslinked to histones. The positions of ${ }^{32} \mathrm{p}$ labeled DNA fragments, crosslinked to different histones and arranged on separate diagonals, were revealed by autoradiography and are indicated by solid lines. The extreme right diagonal (in the upper right corner) is uncrosslinked DNA fragments. The dashed lines show the position in the gel, and the numbers indicate the approximate length (in nucleotides) of ethidium bromide-stained DNA fragments from DNase Idigested rat liver nuclei used as markers. C - "Protein version" of twodimensional gel electrophoresis of ${ }^{123}$ I-labeled histones crosslinked to DNA fragments. The number at each spot indicates the size (in nucleotides) of the DNA fragments crosslinked to histones. For the sake of simplicity, only histone H4 contacts are shown in C. Solid squares indicate the histone-DNA contacts that become attenuated during chromatin unfolding compared with the contacts marked by solid circles. 
be obtained [17]. For example, sea urchin sperm subtypes of histone H2B contain an extended $\mathrm{N}$-terminal domain which reduces the mobility of this histone in the SDS gelelectrophoresis [21]. Lily, as well as other plants, also contains several subtypes of histones $\mathrm{H} 2 \mathrm{~A}$ and $\mathrm{H} 2 \mathrm{~B}$ that are much longer than those from other sources $[22,23]$.

The histone-DNA contacts identified by these crosslinking experiments for isolated core particles [17] was recently confirmed by X-ray crystallography analysis [9] showing that the histone $(\mathrm{H} 3 / \mathrm{H} 4)_{2}$ tetramer is located in the center of the core DNA whereas the two $\mathrm{H} 2 \mathrm{~A} / \mathrm{H} 2 \mathrm{~B}$ dimers flank the tetramer. In addition, the contacts of histone $\mathrm{H} 3$ were located both in the center and at the very end of the core nucleosomal DNA. These sites are very close to each other on the DNA coiled around the histone octamer [17] (Fig. 4). This explained the previous observation that the superhelix of nucleosomal DNA can be stabilized by the histone $(\mathrm{H} 3 / \mathrm{H} 4)_{2}$ tetramer to form a nucleosome-like particle $[24,25]$. Although the contacts of histone $\mathrm{H} 2 \mathrm{~A}$ have similar locations in the center and at the end of the nucleosomal DNA [17], the histone H2A/H2B dimer does not form nucleosomelike particles as found for the $(\mathrm{H} 3 / \mathrm{H} 4)_{2}$ tetramer $[24,25]$. Thus the $(\mathrm{H} 3 / \mathrm{H} 4)_{2}$ tetramer plays an essential role in generating a nucleosome scaffold that is completed by the binding of $\mathrm{H} 2 \mathrm{~A} / \mathrm{H} 2 \mathrm{~B}$ dimers and a linker histone.

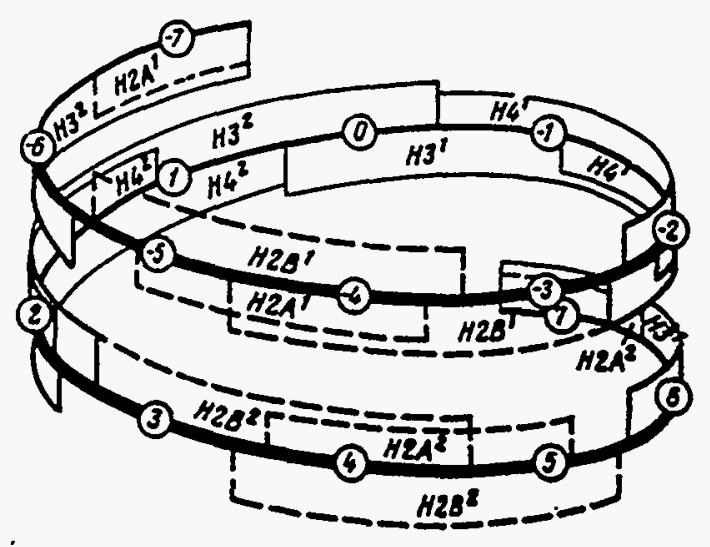

Figure 4. A model for the symmetrical arrangement of histones on the folded core nucleosomal DNA. The DNA forms a left-handed superhelix containing about 80 bp per turn. Positioning of histones H2A (narrow box, broken line), H2B(wide box, broken line), H3 (wide box solid line) and $\mathrm{H4}$ (namrow box, solid line) on both DNA strands is shown above and below the DNA line. Superscripts $l$ and 2 indicate two copies of each histone in the core. The numbers in the open circles represent the number of DNA double helical turns from the dyad axis (indicated by position 0 ), in both directions indicated by + and $-[8]$. 


\section{Conformational Changes in the Nucleosomal Core Revealed by Crosslinking in Linker Histone-Depleted Chromatin}

Protein-DNA crosslinking experiments on intact nuclei isolated from different sources revealed that the sequential arrangement of histone-DNA contacts in the nucleosomal core is very similar. However, it was noticed that the relative strengths of certain histone-DNA contacts in these sources varied and correlated with chromatin activity and compaction [13]. The chromatin in sea urchin sperm is completely inactive in RNA and DNA synthesis and is more densely packed than mitotic chromosomes [26]. In contrast, the chromatin in yeast and in the dividing cells of lily bud sepals is very active in transcription and replication and is significantly or completely depleted of histone $\mathrm{H} 1$ [27-29]. It was found that in the nucleosomal core in sea urchin sperm nuclei (Fig. 3A) contacts $\mathrm{H} 4(57)$ and $\mathrm{H} 4(66)$ have almost equal strength, as do contacts $\mathrm{H} 2 \mathrm{~B}(109)$ and $\mathrm{H} 2 \mathrm{~B}(119)$ (numbers in parenthesis indicate the distance in nucleotides from the 5 '-end of one strand of core nucleosomal DNA to the particular histone contact). However, a different ratio in the intensities of the above-mentioned contacts was found in nucleosomes from lily (Fig. 3B) and yeast (Fig. 3C) nuclei where contact H4(66) was attenuated compared with contact H4(57). A similar ratio in intensities was also found

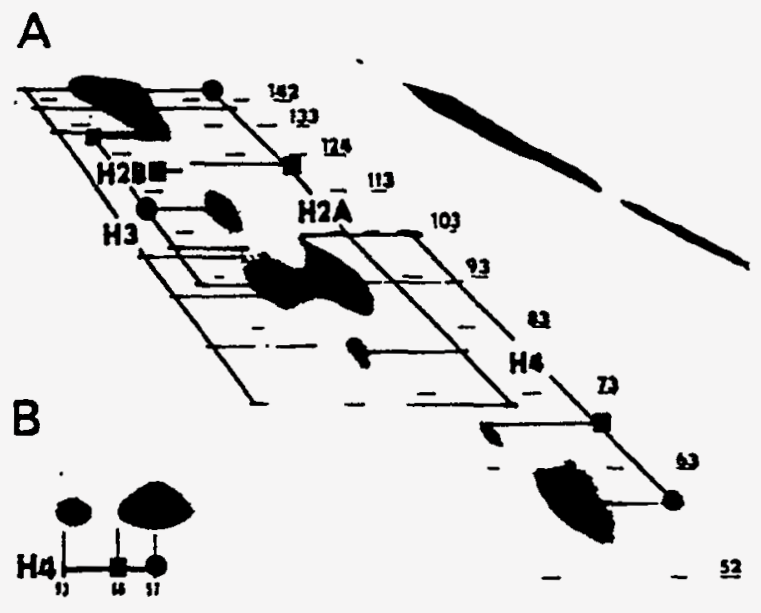

Figure 5. Histone-DNA contacts in chromatin depleted of linker histones. A - "DNA version" of two-dimensional gel electrophoresis of single-stranded, ${ }^{32}$ P-labeled DNA fragments crosslinked to histones in chromatin reconstituted from purified chicken erythrocyte histones and DNA. B - "Protein version" of two-dimensional gel electrophoresis of ${ }^{125}$ I-labeled histones crosslinked to DNA in mouse ascites chromatin. For the sake of simplicity, only histone H4 contacts are shown. For details, see the legend for Fig. 3. 
for the contacts of histone H2B in lily (Fig. 3B) and yeast nuclei, showing attenuation of contacts $\mathrm{H} 2 \mathrm{~B}(119)$ and $\mathrm{H} 2 \mathrm{~B}(129)$ compared with contact $\mathrm{H} 2 \mathrm{~B}(109)$.

Since transcriptionally active chromatin is unfolded and significantly depleted of linker histones, the histone-DNA contacts were also studied in linker histone-depleted chromatin from mouse ascites cells which was used as a hypothetical model of transcriptionally active chromatin. In order to avoid the influence of other factors on nucleosome structure, histone-DNA contacts were also studied in chromatin reconstituted from purified chicken erythrocyte histone octamers and DNA. It is interesting that in both types of linker histone-depleted chromatin (native and reconstituted) the alterations in the contacts of histones $\mathrm{H} 4, \mathrm{H} 2 \mathrm{~A}$ and $\mathrm{H} 2 \mathrm{~B}$ with DNA were the same as in lily and yeast nuclei [13] (Fig. 5). These results suggested that the conformation of the nucleosomal core in linker histone-depleted chromatin is similar to that found in yeast and lily bud sepals nuclei, but is different to that found in sea urchin sperm nuclei. These results also correlate with the unfolded state of both native or reconstituted linker histone depleted chromatins and chromatin in yeast and lily bud sepals nuclei. Indeed, histone $\mathrm{Hl}$ may not be present in significant amounts in nuclei of actively dividing lily cells, where the chromatin is very active in transcription and replication. In yeast, a considerable part of the genome is also unfolded and actively transcribed $[28,29]$. These experiments showed that the level of chromatin compaction correlates with the alterations in histone-DNA contacts in the nucleosomal core and suggests that changes in the level of chromatin compaction lead to gross structural changes in the nucleosome [13].

\section{Conformational Changes in the Nucleosomal Core Caused by Stretching of the Linker DNA During Chromatin Unfolding.}

Because the same core histone-DNA contacts in nuclei from different sources differed in intensity, it was essential to check whether these structural changes were inherent in the nucleosomal cores themselves or were somehow dependent on chromatin structure. For this purpose the histone-DNA contacts were studied in core particles, crosslinked after their isolation from reconstituted chicken erythrocyte chromatin lacking linker histones, from mouse ascites chromatin depleted of linker histones and from yeast nuclei (Fig. 6). It was found that in the core particles isolated from all of these sources, the relative intensities of certain contacts of histones $\mathrm{H} 4, \mathrm{H} 2 \mathrm{~A}$ and $\mathrm{H} 2 \mathrm{~B}$ are similar to those observed in nuclei from sea urchin sperm (Fig. 3A) where the chromatin is highly condensed and repressed. The fact, that the mentioned contacts were restored after the removal of linker DNA, indicates that core histone-DNA contacts are affected by linker DNA.

The chromatin in the nuclei of sea urchin sperm is highly condensed [26] so that the linker DNA is tightly packaged (Fig. 7A) and might not affect the histone-DNA contacts in the nucleosomal core. In contrast, in chromatin depleted of linker histones, which appears as an unfolded filament (Fig. 7B), certain histone-DNA contacts are altered in strength (Fig. 3B,C, Fig. 5) compared with those in nuclei where the chromatin is highly 
condensed (Fig. 3A). However, after removal of linker DNA, for example during core particle isolation (Fig. 7C), the mentioned contacts become as strong (Fig. 6) as in the highly condensed chromatin of sea urchin sperm nuclei (Fig. 3A). Since the strength of the described contacts in nuclei where the chromatin is highly condensed and linker DNA is tightly packaged was found to be the same as for the isolated core particles where the linker DNA is absent, the removal of linker DNA can be considered equivalent to condensation in terms of its influence on the observed histone-DNA contacts in the nucleosomal core. According to these observations it was suggested that

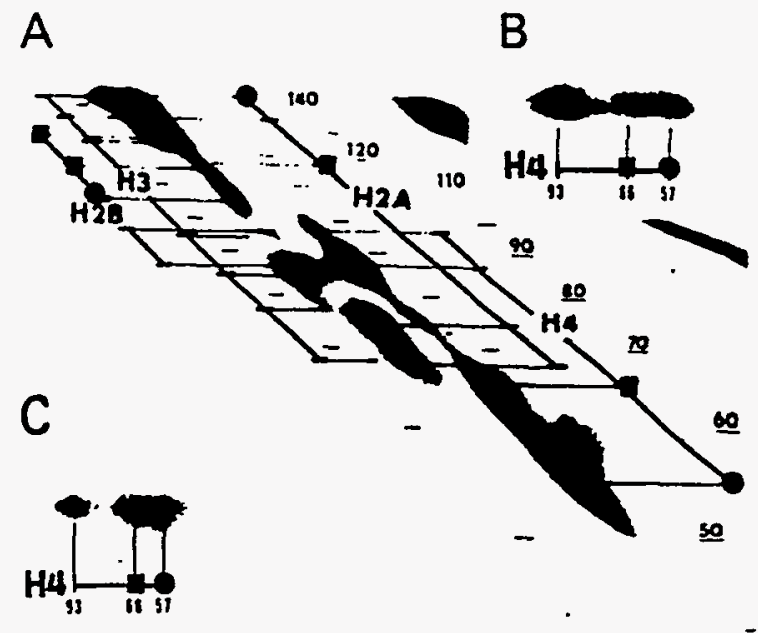

Figure 6. Histone-DNA contacts in core nucleosomes after isolation. A - "DNA version" of two-dimensional gel electrophoresis of single-stranded, ${ }^{32}$ P-labeled DNA fragments crosslinked to histones in core particles isolated from reconstituted chromatin lacking linker histones. B and C "Protein version" of two-dimensional gel electrophoresis of ${ }^{125} \mathrm{I}$ labeled histones crosslinked to DNA in core particles isolated from mouse ascites chromatin depleted of linker histones and from yeast nuclei respectively. For the sake of simplicity, only histone H4 contacts are shown. For details, see the legend for Fig. 3.

certain contacts that exist in highly repressed and condensed chromatin may be distorted when histone $\mathrm{H} 1$ is released and linker DNA is partially unfolded during chromatin activation, but restored again after chromatin condensation. This demonstrates that the alterations in histone-DNA contacts are reversible and depend on the specific arrangement of linker DNA, which could be affected by linker histones. These results suggested that the nucleosomes in linker histone-depleted chromatin might undergo 
some deformation due to the stretching of the linker DNA (Fig. 7). This deformation could cause alterations in the histone $\mathrm{H} 4$ and $\mathrm{H} 2 \mathrm{~A} / \mathrm{H} 2 \mathrm{~B}$ contacts with the sharply bent

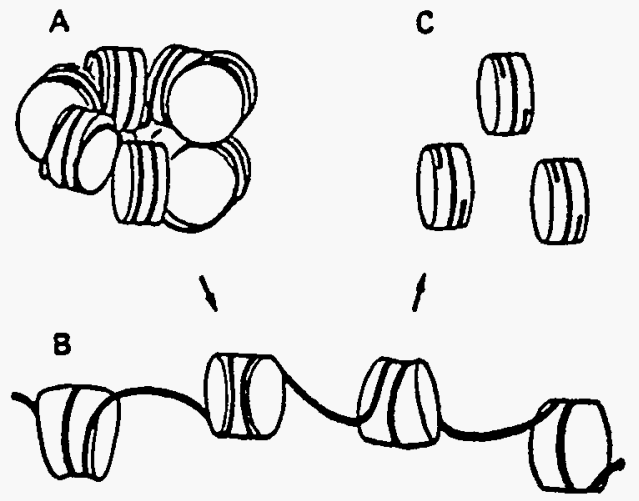

Figure 7. Effect of linker DNA on the conformation of the nucleosomal core. A -hypothetical solenoidal model of $30 \mathrm{~nm}$ chromatin fiber where the linker DNA is tightly packaged as continuation of core nucleosomal DNA as suggested. B - formation of stretched nucleosomes in unfolded chromatin depleted of linker histones. The stretching of the linker DNA causes the deformation (shown by the changed form) of nucleosomal cores. C isolated nucleosomal core particles. Removal of the linker DNA causes the stretched nucleosomes to revert to the conformational state similar to that in highly condensed chromatin in nuclei (A).

regions of nucleosomal DNA, around sites $+/-1,+/-4$ and $+/-5$ respectively (Fig. 8). These alterations were attributed to the structural transition of the nucleosomal core, yielding a new conformational state called a "stretched nucleosome" [13]. The observation of the "stretched nucleosome" in unfolded chromatin from different sources, containing different histone variants with different lengths of $\mathrm{N}$ - and $\mathrm{C}$-terminal domains, suggested that this conformation is very similar for all species studied.

\section{Structural Polymorphism of Nucleosomes in Hyperacetylated and Hypoacetylated Chromosomal Domains}

Genetic and biochemical studies have suggested that the nucleosome itself is involved in the regulation of chromatin activity through the reversible postranslational modifications of histones [30] which have been strongly correlated with chromatin 


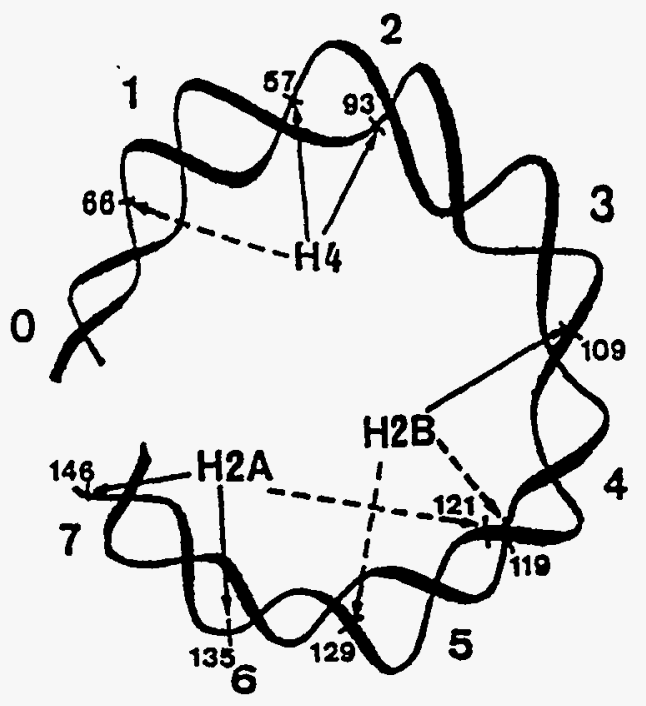

Figure 8. Arrangement of histone-DNA contacts on half of the core nucleosomal DNA for histones $\mathrm{H} 2 \mathrm{~A}, \mathrm{H} 2 \mathrm{~B}$ and $\mathrm{H} 4$. The dashed arrows indicate the aitered contacts. The smaller numbers at the sites of histone-DNA contacts indicate the distance in nucleotides from the S'end of core nucleosomal DNA. The larger numbers on the outside of the helix represent the number of DNA double helical turns from the dyad axis (indicated by position 0 ) [8].

functions required at different phases of the cell cycle [2]. The most extensively studied histone modification associated with chromatin activation is the reversible acetylation of the $\varepsilon$-amino group of specific internal lysine residues located in the flexible and highly basic $N$-terminal domains of core histones $[2,30-34]$. Acetylation neutralizes the positive charges of specific lysines in histones destabilizing their interactions with the negatively charged DNA phosphate groups and thereby can facilitate chromatin decondensation and/or the maintenance of an open conformation in active chromatin regions [2]. Since acetylation is not the only variable factor in the composition of functionally different chromatin regions, it is essential to study the structures of the nucleosomes in these different regions within intact nuclei. This study addresses the structural features of nucleosomes in functionally different chromosomal domains within nuclei from the same cells which contain different levels of histone acetylation and other components e.g. histone $\mathrm{H} 1$ and nonhistone proteins normally present in the nuclear environment. For this purpose histones were crosslinked to DNA within intact nuclei and histone-DNA contacts were mapped in 146 base pairs core particles isolated from 
hyperacetylated, hypoacetylated and nonacetylated chromatin fractions. These crosslinking experiments have detected changes in the patterns of crosslinked peptides in hyperacetylated chromosomal domains versus nonacetylated domains suggesting a restructuring of histone-DNA interactions in these chromatin regions [35]. The affected contacts are formed by the trypsin resistant histones structured domain and by the histone H2A C-terminal domain [36]. None of these histone regions contain sites of acetylation. However, the altered contact $H 4(66)$ due to the crosslinking via His 18 [37], is very close to the site of acetylation, Lys 16. The alteration in this histone $\mathrm{H} 4$ contact is consistent with an observed reduction in the intensity of the crosslinked peptide of histone $\mathrm{H} 4$ in the hyperacetylated chromosomal domain and may be an indication of either loosened binding or rearrangement of the histone $\mathrm{H} 4 \mathrm{~N}$-terminal domain [38]. This provides strong evidence that nucleosomes in these domains are in different conformations. In addition to the high level of acetylation of the core histones, the hyperacetylated chromosomal domain was significantly depleted of linker histone H1. It is interesting that the alterations of core histone-DNA contacts in this domain (38) were very similar to those observed for chromatin depleted of linker histones (Fig. 5) or for the chromatin active in transcription and replication within nuclei from different sources [13] (Fig. 3B, C).

Although the locations of histone tails are undetermined, biochemical experiments in vitro show that acetylation can destabilize their interaction with DNA [39]. However, it is unknown whether acetylation causes the release of histone tail domains or a loosening of their binding in vivo [35]. The fact that most of the altered contacts in the nucleosomal core observed in the crosslinking experiments involve the histone structured regions [36] which are not subject to acetylation suggests that the structural changes occur throughout the nucleosome resulting in an overall different conformation. It should be noted that the contacts identified in the nucleosomal core within the hypoacetylated chromosomal domain were also observed in nuclei from different sources [17] and are fully consistent with observed contacts in the crystal structure of the nucleosome core particle [9]. The contacts affected in the hyperacetylated chromosomal domains, are located close to the sharply bent regions of nucleosomal DNA, around sites $+/-1,+/-4,+/-5$ [9] (Fig. 8) and therefore are expected to be sensitive to the conformational changes which could occur in core nucleosomal DNA during chromatin unfolding induced by histone hyperacetylation and/or $\mathrm{Hl}$ depletion. The coexistence of features such as hyperacetylation of core histones and significant depletion of histone $\mathrm{H} 1$ in the same chromosomal domain strongly argues that this chromatin region is not packaged into a stable higher order structure [40]. Recent crosslinking experiments clearly show that this lack of chromatin compaction, as a result of the depletion of histone H1, leads to conformational changes in the nucleosome [13]. One might expect that in the hyperacetylated chromosomal domain, in addition to the deficiency of histone $\mathrm{HL}$, the high level of histone acetylation would cause additional changes in the analyzed histone-DNA contacts. However, repeated crosslinking experiments in intact nuclei did not reveal any additional alterations in the analyzed histone-DNA contacts in the hyperacetylated chromosomal domains [38] compared to those observed recently in bulk H1-depleted chromatin [13]. The alterations in the hyperacetylated chromosomal 
domain could be the result of significant depletion of histone $\mathrm{Hl}$ or the result of core histone acetylation affecting the ability of histone $\mathrm{H} 1$ to condense the chromatin [41, 42] or both, such that the hyperacetylated chromosomal domain is maintained in a less folded state than the hypoacetylated or nonacetylated domains.

\section{Stretching of the Linker DNA During Chromatin Unfolding Causes the Conformational Changes in the Nucleosomal DNA}

Transcriptionally active chromatin is unfolded and significantly depleted of linker histones [43], which play a key role in chromatin compaction $[44,45]$. The binding of histone HI to linker DNA considerably reduces its electrostatic free energy which determines linker DNA bending during chromatin folding into the higher order structure [46]. In contrast, in linker histone depleted chromatin the distance between the points where the linker DNA enters and leaves the nucleosome core may be increased by repulsion between adjacent linker DNA segments $[44,47-50]$ and thereby can affect the conformation of the nucleosome core DNA. To address the question of whether or not the alterations in histone-DNA contacts during chromatin unfolding, which were observed in H1-depleted chromatin [13] and in the hyperacetylated chromosomal domains [38] are caused by changes in nucleosome core DNA conformation, the histone-DNA contacts have been analyzed in isolated core particles under ionic conditions that affect DNA stiffness.

Several physical and biochemical studies have demonstrated that a reduction of the monovalent ion concentration below $10 \mathrm{mM}$ has a significant effect on the nucleosome core structure (reviewed in [3] ). It has also been well documented that the loss of counterions at low ionic strengths increases the electrostatic repulsion of unneutralized DNA phosphate groups resulting in an increase in DNA stiffness [51]. This increase in stiffness may cause stretching of the nucleosomal DNA which in turn may change the conformation of the nucleosome [52] and thereby affect histone-DNA contacts.

Crosslinking experiments showed that the alterations in histone-DNA contacts in isolated core particles induced at low ionic strength are caused by a decrease in the neutralization of negative DNA charges [14] and are identical to those observed in nucleosomes in H1-depleted chromatin [13] and in hyperacetylated chromosomal domain [38]. It was suggested that the conformational changes in nucleosome core particles at low ionic strength and in nucleosomes during chromatin unfolding were due to the stretching of the nucleosome core DNA [14]. Based on these observations a model for the reversible nucleosome structural transition during chromatin unfolding was proposed (Fig. 9). This model demonstrates how the nucleosome structure is changed at the first step of chromatin activation, chromatin decondensation and suggests that these changes may facilitate the second step, which involves either remodeling or removal of the nucleosome. It also suggests that the lack of chromatin compaction in the hyperacetylated chromosomal domain can cause similar structural changes in the nucleosomal DNA yielding a new nucleosome conformation. 


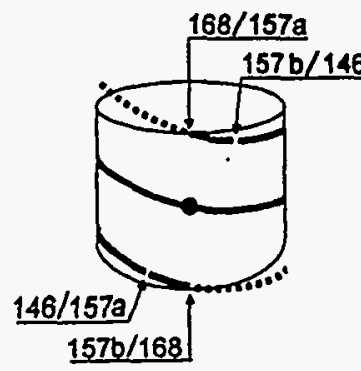

A

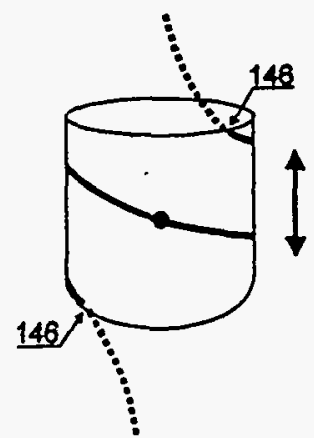

B

\begin{abstract}
Figure 9. Schematic representation of the reversible formation of a "stretched nucleosome". The change in the nucleosomal DNA conformation due to its stretching causes a release from the histone octamer of approximately 10 bp of DNA at each side. Arrows with numbers indicate the sites of preferential microcoecal nuclease cleavage yielding nucleosome particles with 146, 157 and 168 bp DNA. The nucleosomal DNA terminal fragments in the compact form of the nucleosome (A) slide off of the histone octamer and turn into a linker DNA (represented by a dashed line) in the "stretched nucleosome" (B). Arrows on the right side show the direction of the stretching resulting in a prolate shape of the nucleosome.
\end{abstract}

\title{
9. Location of the Histone H2A C-Terminal Domain in the Nucleosome
}

Current information about the nucleosome structure is derived from data about the arrangement of histone structured globular domains. However, there is no information about the location of histone terminal domains in the nucleosome. These unstructured histone terminal domains have been shown to participate in the folding of oligonucleosomes [53] and in the stabilization of higher order chromatin structure [54]. In addition they contain sites for reversible postranslational modifications which occur during the different phases of the cell cycle and correlate with chromatin functions. The question of how exactly these modifications are involved in this regulation still remains unclear. One way to understand the functional role of these important histone regions is to determine their locations and to observe their behavior during chromatin functions. The only histone-terminal domain localized in the nucleosome so far is the histone $\mathrm{H} 2 \mathrm{~A}$ C-terminal domain [38]. For these purposes zero-length covalent protein-DNA crosslinking was used to map histone-DNA contacts in nucleosome core particles from which histone-terminal domains were selectively trimmed by trypsin or clostripain. It was found that compared to the intact (control) core particle (Fig. 10A) the contact H2A at the dyad axis (nucleotide 77) was heavily attenuated in core particles digested with trypsin (Fig. 10B) which trims both $\mathrm{N}$ - and C-terminal domain of histone H2A [55]. 
However this contact did not change in core particles digested with clostripain (Fig. 10D) which trims only $\mathrm{N}$-terminal tail of histone H2A leaving its $\mathrm{C}$-terminal tail intact [56]. Since all other contacts of histone H2A at the end of nucleosomal DNA were not affected (Fig. 10C), this indicates that the trypsin-sensitive $\mathrm{C}$-terminal domain of histone H2A makes a contact with the DNA at the nucleosome dyad axis while its globular domain interacts mostly with the end of core nucleosomal DNA (Fig. 11).

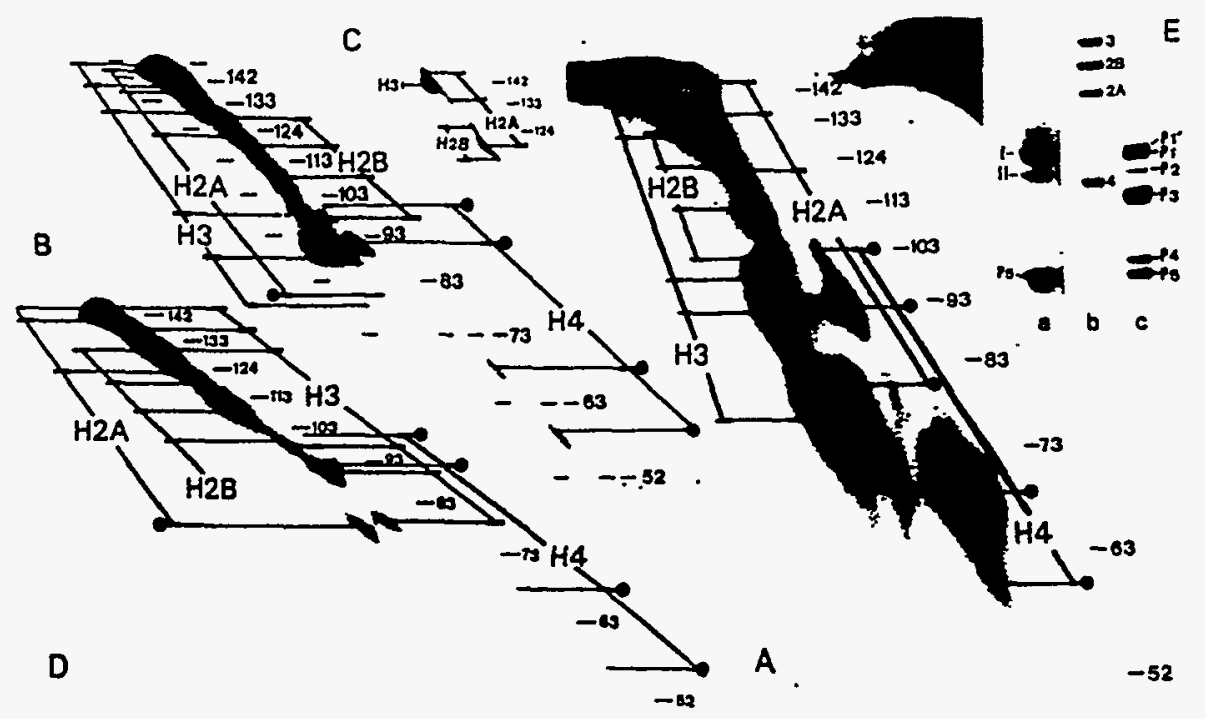

Figure 10. "DNA version" of two-dimensional gel electrophoresis of single-stranded, ${ }^{32} \mathrm{p}$-labeled DNA fragments crosslinked to histones in core particles. A - Intact (control) core particles; B - Trypsin-digested core particles; C - Shorter exposure of the upper part of B; D - Clostripain-digested core particles. E - SDSelectrophoresis of peptides from clostipain (lane a)- and trypsin (lane b)-digested core particles and histones (lane b) from intact (control) core particles. Bands I and II (lane a in E) correspond to peptides of histone H2A/H2B and H3 respectively. Peptides P1/P1', P2 and P3 comespond to histones H3, H2A and H2B respectively. Solid circles indicate the signals which are heavily attenuated in trypsin (B)- or clostripain (D)- digested core particles compared to the intact (A) core particles. For other details see the legend for Fig. 3.

It should be mentioned that the nucleosomal DNA deviates from its superhelical path at the dyad axis. This deviation was abolished in H1-depleted chromatin [57] which is consistent with conformational changes in the nucleosomal DNA during chromatin unfolding as revealed by histone-DNA crosslinking [13]. The contact of histone H2A at the dyad axis that is detected due to the crosslinking of histone H2A C-terminal domain [36] via His-123 [58], was not observed in nuclei from sea urchin sperm, lily bud sepals and yeast [17] (Fig. 3) in which the histone H2A C-terminal domain does not contain this histidine [59-61]. This is the reason why it was thought previously that histone H2A does not make this contact in nuclei but only in isolated core particles [17]. The Hela 
histone $\mathrm{H} 2 \mathrm{~A}$ C-terminal domain, however, contains two histidines at positions 123 and 124 [62] and it was found that in nuclei this domain makes a very strong contact at the dyad axis in the hypoacetylated chromosomal domain, whereas in the hyperacetylated domain this contact was absent [38]. Because the histone H2A C-terminal domain is not acetylated, this suggests that the absence of the histone $\mathrm{H} 2 \mathrm{~A}$ contact with the dyad axis in the hyperacetylated domain is caused by conformational changes in nucleosomal DNA at this site [57] and is not, as was thought before, the result of the rearrangement of histone H2A C-terminal domain [36]. The observed conformational changes may be

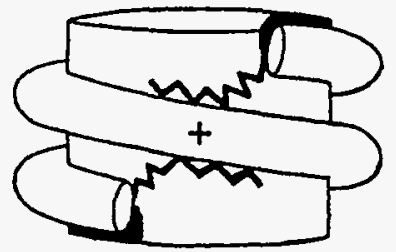

Figure 11. Model for the arrangement of histone $\mathrm{H} 2 \mathrm{~A}$ globular and C-terminal domain. Black solid region indicates the globular domain and zigzag lane indicates the $\mathrm{C}$-terminal domain of histone H2A. The plus sign shows the nucleosomal dyad axis (at nucleotide 77 ). passing through the nucleosome perpendicular to the plane of the figure.

also the result of a significant depletion of histone $\mathrm{H} 1$ in the hyperacetylated chromosomal domain which is supported by the recent finding of the loss of the same contact in bulk H1-depleted chromatin [13] and by the observed loss of the H2A crosslinked peptide in the protein pattern of the acetylated chromosomal domain [35]. These different conformations of the nucleosome observed as changes in histone-DNA contacts in the active chromatin regions may play a biological role rendering the nucleosome competent for transcription and/or replication.

\section{Summary}

It is now clear that the nucleosome as a functional unit is involved in the modulation of chromatin structure/function relationships through alterations in histone-DNA contacts. One of the ways this regulation may occur is through reversible posttranslational modifications of histones associated with different phases of the cell cycle. Since the sites for these modifications are located in the flexible and unstructured histone terminal 
domains, the localization of these histone regions in the nucleosome and the observation of their arrangement through the cell cycle is very important for our understanding of their function. Only the C-terminal domain of histone H2A contains histidine which is a protein crosslinking site. The localization of this histone terminal domain in the nucleosome [36] clearly demonstrates the ability of the crosslinking methodology to determine the location of other histone terminal domains on the nucleosomal DNA. For example, using the method of site directed mutagenesis in higher eukaryotic cells, it is possible to insert histidines into other histone terminal domains for the localization of these important histone regions on the nucleosomal DNA by protein-DNA crosslinking. This will allow the observation of their arrangement during the cell cycle and elucidate the role of these histone regions in nucleosome functions.

\section{Acknowledgements}

This work was supported by a grant from the Department of Energy (DE-FGO388ER60673).

\section{References}

1. Saitoh, Y., Laemmli, U. K. (1993) From the chromosomal loops and the scaffold to the classic bands of metaphase chromosomes, Cold Spring Harbor Symposia on Quantitative Biology 58, 755-765.

2. Bradbury, E. M. (1992) Reversible histone modifications and the chromosome cell cycle, Bioessays 14, 916.

3. Van Holde, K. (1988) Chromatin, Springer-Verlag, New York, Berlin, Heidelberg, London, Paris, Tokyo.

4. Arents, G., Burlingarne, R. W., Wang, B. C., Love, W. E., and Moudrianakis, E. N. (1991) The nucleosomal core histone octamer at $3.1 \AA$ resolution: a tripartite protein assembly and a left-handed superhelix, Proc. Nath. Acad. Sci. USA 88, 10148-10152.

5. Suau, P., Kneale, G. G., Braddock, G. W., Baldwin, J. P., and Bradbury, E. M. (1977) A low resolution model for the chromatin core particle by neutron scattering, Nucl. Acids Res. 4, 3769-3786.

6. Finch, J. T., Brown, R. S., Rhoddes, D., Richmond, T., Rushton, B., Lutter, L. C., and Klug, A. (1981) Xray-diffraction study of a new crystal form of the nucleosome core showing higher resolution. J. Mol. Biol. 145, 757-769.

7. Bentley, C. A. Finch, J. T., and Lewit-Bentley, A. (1981) Neutron diffraction studies on crystals of nucleosome cores using contrast variation, J. Mol. Biol. 145, 771-784.

8. Richmond, T. J., Finch, J. T., Rushton, B., Rhodes, D., and Klug. A.(1984) Structure of the nucleosome core particle at 7 A resolution, Nature 311, 532-537.

9. Luger, K., Mader, A. W., Richroond, R. K., Sargent, D. F., and Richmond, T. J. (1997) Crystal structure of the nucleosome core particle at 2.8 A resolution, Nature 389, 251-260.

10. Wood, M. J., Yau, P.M., Imai, B. S., Goldberg, M. W., Lambert, S. J., Fowler, A. L., Baldwin, J. P., Godfrey, J., Moudrianakis, E. N., Ibel, K., May, R. P., Koch, M., and Bradbury, E. M. (1991) Neutron and $x$-ray scatter studies of the histone octamer and amino and carboxyl domain trimmed octamers, $J$. Biol. Chem. 266, 5696-5702.

11. Schroth, G. P., Yau, P. M., Imai, B. S., Gatewood, J. M., and Bradbury, E. M. (1990) A NMR study of mobility in the histone octamer, FEBS Lett. 268, 117-120.

12. Levina, E. S., Barykin, S. G., Shick, V. V., and Mirzabekov, A. D. (1981) The method of crosslinking histones to DNA partly depurinated at neutral pH, Anal. Biochem. 110, 93-101. 
13. Usachenko, S. I., Gavin, L. M., and Bavykin, S. G. (1996) Alterations in nucleosome core structure in linker histone-depleted chromatin, J. Biol. Chem. 271, 3831-3836.

14. Gavin, I. M., Usachenko, S. L., and Bavykin, S. G. (1998) Nucleosome structural transition during chromatin unfolding is caused by conformational changes in nucleosomal DNA, J. Biol. Chem. 273, 2429-2434.

15. Karpov, V. L., Preobrazhenskaya, O. V., and Mirzabekov, A. D. (1984) Chromatin structure of hsp 70 genes, activated by heat shock: selective removal of histones from the coding region and their absence from the 5' region, Cell 36, 423-431.

16. Bavykin,S.. Srebreva, L.. Banchev, T.. Tsanev, R., Zlatanova, J., and Mirzabekov, A. (1993) Histone HI deposition and histone-DNA interactions in replicating chromatin, Proc. Natl. Acad. Sci. USA 90, 39183922.

17. Bavykin. S. G., Usachenko, S. I., Lishanskaya, A. I., Shick, V. V., Belyavsky, A. V., Undritsov, I. M., Strokov, A. A., Zalenskaya, I. A., and Mirzabekov, A. D. (1985) Primary organization of nucleosomal core particles is invariable in repressed and active nuclei from animal, plant and yeast cells, Nucl. Acids Res.13, 3439-3459.

18. Nacheva, G. A.. Guschin, D. Y., Preobrazhenskaya, O. V., Karpov, V. L., Ebralidse, K. K. and Mirzabekov, A. D. (1989) Change in the pattern of histone binding to DNA upon transcriptional activation, Cell 58, 27-36.

19. Shick, V. V., Belyavsky, A. V., Bavykin, S. G., and Mirzabekov, A. D. (1980) Primary organization of the nucleosome core particles, J. Mol. Biol. 139, 491-517.

20. Belyavsky, A. V., Bavykin, S. G., Goguadze, E. G., and Mirzabekov, A. D. (1980) Primary organization of nucleosomes containing afl five histones and DNA 175 and 165 base-pairs long, J. Mol. Biol. 139, 519. 536.

21. Strickland, M., Strickland, W. N., Brandt, W. F., Von Hoit, C., Wittmann-Liebold, B., Lehmann. A. (1978) The complete amino-acid sequence of histone H2B from sperm of the sea urchin Parechinus angulosus, Eur. J. Biochem. 89, 443-452.

22. Spiker. S. (1975) An evolutionary comparison of plant histones, Biochim. Biophys. Acta 400, 461-467.

23. Spiker, S., Key, J. L. and Wakim, B. (1976) Identification and fractionation of plant histones, Arch. Blochem. Biophys. 176, 510-518.

24. Moss, T., Stephens, R. M., Crane-Robinson, C., and Bradbury, E. M. (1977) A nucleosome-like structure containing DNA and the arginine-rich histones H3, H4, Nucl. Acids Res. 4, 2477-2485.

25. Thomas, J. O., and Oudet, P. (1979) Complexes of the arginine-rich histone tetramer $(\mathrm{H} 3)_{2}(\mathrm{H} 4)_{2}$ with negatively supercoiled DNA: electron microscopy and chemical cross-linking, Nucl. Acids Res. 7, 611623.

26. Poccia, D. L., and Green, G. R. (1992) Packaging and unpackaging the sea urchin sperm genome, Trends Biochem.17, 223-227.

27. Paranjape, S. M. Kamakaka, R. T., and Kadonaga, J. T. (1994) Role of Chromatin Structure in the Regulation of Transcription by RNA Polymerase II, Ansu. Rev. Biochem. 63, 265-297.

28. Lohr, D. and Hereford, L (1979) Yeast chromatin is uniformly digested by DNase L, Proc. Nath. Acad. Sci. USA 76, 4285-4288.

29. Hereford, L. M. and Rosbash, M. (1977) Number and distribution of polyadenylated RNA sequences in yeast, Cell 10, 453-462.

30. Grunstein, M. (1990) Nucleosomes: Regulators of Transcription, Trends Gen. 6, 395-400.

31. Csordas, A. (1990) On the biological role of histone acetylation, Biochem. J. 265, $23-38$.

32. Davie, J. R. and Hendzel, M. J. (1994) Multiple functions of dynamic histone acetylation, J. Cell Biochem. 55, 98-105.

33. Tumer, B. M. (1991) Histone acetylation and control of gene expression, J. Cell Sci. 99, 13-20.

34. Loidl, P. (1994) Histone acetylation: facts and questions, Chromosoma 103, 441-449.

35. Ebralidse, K. K. Hebbes, T. R., Clayton, A. L. Thome, A. W., and Crane-Robinson, C. (1993) Nucleosomal structure at hyperacetylated loci probed in nuclei by DNA-histone crosslinking, Nucl. Acids Res. 21, 4734-4738.

36. Usachenko, S. 1, Bavykin, S. G., Gavin, L. M., and Bradbury, E. M. (1994) Rearrangement of the histone H2A C-terminal domain in the nucleosome, Proc. Nath Acad. Sci. USA 91, 6845-6849. 
37. Ebralidse, K. K., Grachev, S. A., and Mirzabekov, A. D. (1988) A highly basic histone H4 domain bound to the sharply bent region of nucleosomal DNA, Nature 331, 365-367.

38. Usachenko, S. l., and Bradbury, E. M., manuscript in preparation.

39. Hong, L., Schroth, G. P., Matthews, H. R., Yau, P., and Bradbury, E. M. (1993) Studies of the DNA binding properties of histone $H 4$ amino terminus, J. Biol. Chem. 268, 305.314.

40. Van Holde, K. E; and Zlatanova, J. (1996) What determines the folding of the chromatin fiber?, Proc. Natl. Acad. Sci. USA 93, 10548-10555.

4I. Ridsdale, J. A., Hendzel, M. J., Delcuve, G. P., and Davie, J. R. (1990) Histone acetylation alters the capacity of the $\mathrm{H} l$ histones to condense transcriptionally active/competent chromatin, J. Biol. Chem. 265, 5150-5156.

42. Perry, C. A. and Annunziato, A. T. (1991) Histone acetylation reduces H1-mediated nucleosome interactions during chromatin assembly, Exp. Cell Res. 196, 337-345.

43. Zlatanova, J., and Van Holde, K. (1992) Histone Hl and transcription: still an enigma?, J. Cell Sci. 103, 889-895.

44. Thoma, F., Koller, Th., and Klug, A. (1979) involvement of histone $H 1$ in the organization of the nucleosome and of the salt-dependent superstructures of chromatin, J. Cell Biol. 83, 403-427.

45. Widom, J. (1989) Toward a unified model of chromatin folding, Annu. Rev. Biophys. Chem. 18, 365-395.

46. Clark, D. J., and Kimura, T. (1990) Electrostatic mechanism of chromatin folding, J. Mol. Biol. 211, 883896.

47. Goulet, I., Zivanovic, Y., Prunell, A., and Revet, B. (1988) Chromatin reconstitution on small DNA rings, J. Mol. Bial. 200, 253-266.

48. Zivanovic, Y., Duban-Goulet, L., Schultz, P., Stofer, E., Oudet, P., and Prunell, A. (1990) Chromatin reconstituted on small DNA rings. Histone HS dependence of DNA supercoiling in the nucleosome, $J$. Mol. Biol. 214, 479-495.

49. Bednar, J., Horowite, R. A., Dubochet, J., and Woodcock, C. L. (1995) Chromatin conformation and saltinduced compaction: three-dimensional structural information from cryoelectron microscopy, J. Cell Biol. 131, 1365-1376.

50. Hamiche, A. Schultz, P., Ramakrishnan, V., Oudet, P., and Prunell, A. (1996) Linker histone-dependent DNA structure in liniar mononucleosomes, J. Mol. Biol. 257, 30-42.

51. Manning, G. S. (1978) The molecular theory of polyelectrolyte solutions with applications to the electrostatic properties of polynucleotides, $Q$. Rev. Biophys. 11, 179-246.

52. Marky, N. L., and Manning, G. S. (1991) The elastic resilience of DNA can induce all-or-none structural transitions in the nucleosome core particle, Biopolymers 31, 1543.1557.

53. Garcia-Ramirez, M., Dong, F., and Ausio, J. (1992) Role of the histone "tails" in the folding of oligonucleosomes depleted of histone HI, J. Biol. Chem. 267, 19587-19595.

54. Allan, J., Habome, N., Rau, D. C., Gould, H. (1982) Participation of core histone "tails" in the stabilization of the chromatin solenoid, J.Cell Biol. 93, 285-297.

55. Bohm, L. Crane-Robinson, C., and Sautiere, P. (1980) Proteolytic digestion studies of chromatin corehistone structure, Eur. J. Biochem 106, 525-530.

56. Dumuis-Kervabon, A., Encontre, I., Etienne, G., Jauregui-Adell, J., Mery, J., Mesnier, D., and Parello, J. (1986) A chromatin core particle obtained by selective cleavage of histones by clostripain, EMBO J. S, 1735-1742.

57. Pehrson, J. R. (1989) Thymine dimer formation as a probe of the path of DNA in and between nucleosomes in intact chromatin, Proc. NatL. Acad. Sci. USA 86, 9149-9153.

58. Guschin, D. Y., Ebralidse, K. K. and Mirzabekov, A. D. (1991) Peptidic points of H2A, H2B - DNA interactions in the nueleosome core particles, Mol. Biol. (USSR) 25, 1400-1411.

59. Strickland, W. N., Strickland, M. S., de Groot, P. C., and von Holt, C. (1980) The primary structure of histone H2A from the sperm cell of the sea urchin Parechinus angulosus, Eur. J. Biochem. 109, 151-158.

60. Rodrigues, J. de A., Brandt. W. F., and von Holt, C. (1985) The amino acid sequence of wheat histone H2A(1). A core histone with a C-terminal extention, Eur. J. Biochem. 150, 499-505.

61. Choe, J., Kolodrubetz, D., and Grunstein, M. (1982) The two yeast histone H2A genes encode similar protein subtypes, Proc. Natl. Acad. Sci. USA 79, 1484-1487.

62. Zhong, R., Roeder, R. G., Heintz, N. (1983) The primary structure and expression of four cloned human histone genes, Nucl. Acids Res. 11, 7409-7425. 